\title{
Lignification in Relation to the Influence of Water-deficit Stress in Brassica napus
}

\author{
Bok-Rye Lee, Qian Zhang and Tae-Hwan Kim* \\ Department of Animal Science, Institute of Agriculture Science and Technology, College of Agriculture \& Life Science, \\ Chonnam National University, Gwangju 500-757, Korea
}

\begin{abstract}
To investigate lignification process and its physiological significance under water-deficit condition, the responses of peroxidases, polyphenol oxidase (PPO) and phenylalanine ammonia-lyase (PAL) in relation to leaf water status to the short term of water deficit treatment in the leaves with different maturities in forage rape were measured. The significant decrease in relative water content (RWC) and leaf osmotic potential $(\Psi \pi)$ were apparent after $5 \mathrm{~d}$ of water-deficit treatment. The activity of guaiacol peroxidase (GPOD), ascorbate peroxidase (APOD), coniferyl alcohol peroxidase (CPOD), and syringaldazine peroxidase (SPOD) was depressed especially in middle and old leaves when compared with that of control leaves. On the other hand, in young leaves, a significant increase in CPOD $(+34 \%)$ and SPOD $(+24 \%)$ activity as affected by water-deficit treatment was apparent. The activation of PAL and PPO was observed in middle and old leaves for PAL and in young and middle leaves for PPO. These results suggest that peroxidases in middle and old leaves did not involve in lignification under mild water-deficit stress, whereas CPOD and SPOD in young leaves participate in lignification by a coordination with PAL and PPO to incorporate phenol and lignin into the cell walls. (Key words : Brassica napus, Lignification, Peroxdases, Phenylalanine ammonia-lyase, Polyphenol oxidase, Water-deficit stress)
\end{abstract}

\section{I . INTRODUCTION}

Drought occurs when a region receives consistently below average precipitation. When soil conditions and erosion cause a shortage in water usefulness to the crops, the drought condition can be also occurred individually from changes in precipitation levels. This global phenomenon has a widespread impact on ecosystem and agriculture and the affected region is expanding during recent decades.

When the plants are exposed to prolonged periods of drought, the decrease in water availability for transportassociated processes leads to changes in the concentrations of many metabolites, followed by disturbances in protein and carbohydrate metabolism. In previous researches in white clover, it has been established that drought results in an inhibition of de novo protein synthesis (Kim et al., 2004; Lee et al., 2005), which in turn leads to a transient amino acid accumulation(Lee et al., 2005) and an increase in ammonia and compatible solutes such as soluble sugars and proline (Lee et al., 2005; Lee et al., 2008a). The increased proline loading to phloem caused by drought has significance on the down-regulation of $\mathrm{N}$ uptake and the assimilation of newly absorbed $\mathrm{N}$ with decreasing nitrate reductase activity in roots (Lee et al., 2009a). Moreover, in many higher plants under drought stressed condition, the alteration of carbohydrate metabolism in response to drought stress has been reported (Souza et al., 2004). Drought results in an increase of soluble carbohydrate pool and this accumulation is mainly due to the hydrolysis of previously stored starch rather than to de novo synthesis (Lee et al., 2008a). The accumulation of sugars would be involved in dehydration tolerance by protecting the macromolecules through the maintenance of $\mathrm{s}$ sufficient degree of hydration during water loss (Sánchez et al., 2004).

One of the potential reactions of plants to environmental stress including drought is an increased synthesis of specific

\footnotetext{
* Corresponding author: Tae-Hwan Kim, Department of Animal Science, Institute of Agriculture Science and Technology, College of Agriculture \& Life Science, Chonnam National University, Gwangju 500-757, Korea. Tel: +82-62-530-2126, Fax: +82-62-530-2129, E-mail: grass1@chonnam.ac.kr
} 
peptide and proteins. Defense proteins including antioxidant enzymes and pathogenesis related (PR) proteins are induced under stressed condition. Drought induces oxidative stress as evidenced by the increase in reactive oxygen species (ROS) such as $\mathrm{O}_{2}{ }^{-}$and $\mathrm{H}_{2} \mathrm{O}_{2}$ and lipid peroxidation level. Activation of superoxide dismutase (SOD) and catalase (CAT) by drought treatment continued when leaf water potential is above $-1.65 \mathrm{MPa}$ and then their activity remain on the similar level whereas guaiacol peroxidase (GPOD) is increased mostly under progressive drought stress and correlated with increase in lipid peroxidation and growth restriction (Lee et al., 2009b). A close relationship between the increase in PR-proteins activity and the decrease in leaf water potential has been also observed in drought stressed white clover. The enhanced activity of $\beta$-1,3-glucanase, chitinase and cellulase for the early period might be an act of transient tolerance to drought stress, but the activation of these enzymes during terminal stress might be a drought stress-induced injurious symptom (Lee et al., 2008b).

It has been suggested that mild drought improves nutrient value of forage crops. This suggestion would be associated with a transient accumulation of amino acids and soluble carbohydrates only for the early period (Lee et al., 2005; Lee et al., 2008a) when the crops are exposed to mild water deficit stress. However, it has been well documented that severe drought stress is responsible for the increase in cell wall lignification (Katerji et al., 1997). The incorporation of lignin into cell walls results in structural rigidity and durability of plant tissues. Lignin, a component of acid detergent fiber, has been shown to be negatively correlated with the expansion growth and the digestibility in forage crops. Indeed, the kinetics of peroxidases and lignificationrelated enzymes responds to the intensity of drought stress along with the increase of lipid peroxidation and lignin content (Lee et al., 2007). However, little has been known about the responses of peroxidases, polyphenol oxidase (PPO) and phenylalanine ammonia-lyase (PAL), which involve in the pathway of phenolics and lignin synthesis (Rivero et al., 2001), to drought treatment in forage rape plants. In this study, thus, the responses of peroxidases, PPO and PAL in relation to leaf water status to the short term of water deficit treatment in the leaves with different maturities in forage rape.

\section{П. MATERIALS AND METHODS}

\section{Plant culture and sampling}

Twelve-week-old plants (Brassica napus $\quad$ L. cv. Pollen) were exposed to water-deficit stress by decreasing the volume of water supply per day. Daily irrigation of 200 or $20 \mathrm{~mL}$ of water per pot was applied to the well-watered (control) and water-deficit treatment, respectively (Kim et al., 2004). Each treatment lasted for $5 \mathrm{~d}$ and leaf tissues were sampled just before the irrigation at 10:00 am. Tissue samples were immediately frozen in liquid nitrogen. Freezedried samples were finely ground and stored under vacuum for further analysis.

\section{Measurements of leaf water parameters}

Relative water content (RWC) was determined gravimetrically as described previously (Kim et al., 2004). Leaves were collected and frozen in liquid nitrogen. Frozen samples were then allowed to thaw before being centrifuged at $13,000 \times \mathrm{g}$ for $15 \mathrm{~min}$. The osmolality of the collected sap was measured with a vapor pressure osmometer (Lee et al., 2013).

\section{Measurement of antioxidant enzyme activities}

For extraction of enzymes, fresh samples $(0.5 \mathrm{~g})$ were

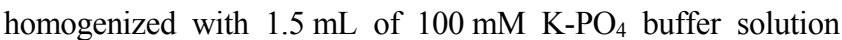
(pH 7.0) containing $2 \mathrm{mM}$ phenylmethylsulfonyl fluoride (PMSF), and centrifuged at $14,000 \times \mathrm{g}$ at $4^{\circ} \mathrm{C}$ for $20 \mathrm{~min}$. The enzyme activity was measured as described by Lee et al. (2007). POD activity with ascorbate as hydrogen donor (ascorbate-peroxidase; APOD) was determined by measuring the decrease in absorbance at $290 \mathrm{~nm}$ (extinction coefficient of $2.8 \mathrm{mM}^{-1} \mathrm{~cm}^{-1}$ ). One unit of enzyme activity was defined as the amount of enzyme that causes the formation of $1 \mu \mathrm{M}$ ascorbate oxidized per min. For guaiacol-peroxidase (GPOD) activity, the oxidation of guaiacol was estimated by measuring the increase in absorbance at $470 \mathrm{~nm}$ for 1 min and the activity was calculated using an absorption for tetraguaiacol (26.6 mM $\left.\mathrm{mm}^{-1} \mathrm{~cm}^{-1}\right)$. One unit of enzyme activity was defined as the amount of enzyme that causes 
the formation of $1 \mu \mathrm{M}$ tetraguaiacol per min. The activity of coniferyl alcohol peroxidase (CPOD) was determined by measuring the decrease in absorbance at $260 \mathrm{~nm}\left(2.21 \mathrm{mM}^{-1}\right.$ $\mathrm{cm}^{-1}$ ). One unit of enzyme activity was defined as the amount of enzyme that causes the formation of $1 \mathrm{mmol}$ coniferyl alcohol oxidized per min. syringaldazine peroxidase (SPOD) activity was determined following the absorbance increase at $530 \mathrm{~nm}\left(27 \mathrm{mM}^{-1} \mathrm{~cm}^{-1}\right)$. One unit of enzyme activity was expressed as $\mu \mathrm{mol}$ syringaldazine oxidized per $\min$.

\section{Determination of lignification-related enzyme activity, lignin and total phenol}

Polyphenol oxidase (PPO) activity was determined according to the method of Siriphanich and Kader (1985). Phenylalanine ammonia-lyase (PAL) activity was assayed by using an assay modified from D'Cunha et al. (1996). The amount of lignin was calculated from a linear calibration curve $(0 \sim 40 \mu$ g) built with commercial alkali lignin (Aldrich, Steinheim, Germany). Total phenol content was determined by FolinCiocalteau method (Singleton and Rossi, 1965). The phenolic contents were estimated using a standard curve using gallic acid. Total phenolic content was expressed as gallic acid equivalents in milligrams per gram fresh weight (Lee et al., 2007).

\section{Statistical analysis}

A completely randomized design was used with three replicates for two water treatments. Tukey's studentized range test was used to compare the means of separate replicates. Unless otherwise stated, conclusions are based on differences between means significant at $\mathrm{p}=0.05$.

\section{RESULTS AND DISCUSSION}

The significant decreases in relative water content (RWC) and leaf osmotic potential $(\Psi \pi)$ were apparent after $5 \mathrm{~d}$ of water-deficit treatment (Fig. 1). The RWC and $\Psi \pi$ in waterdeficit leaves were reached $59.7 \%$ and -1.702 , respectively. These values were slightly less, whereas the rate of decrease in both parameters was relatively higher than those

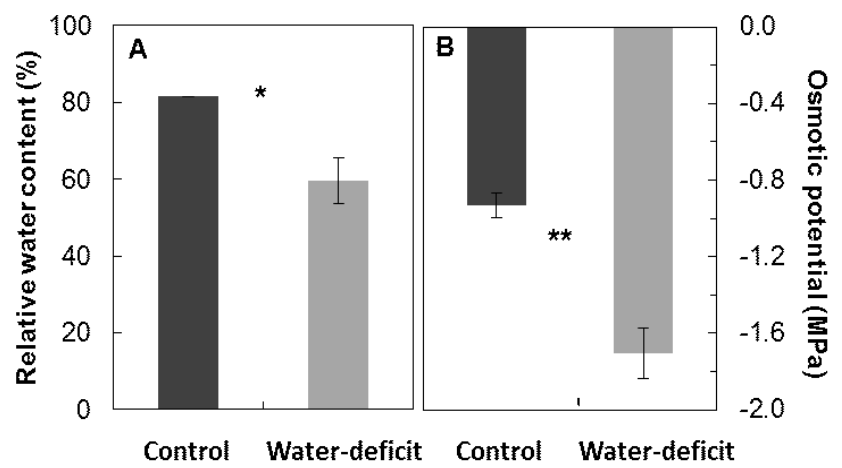

Fig. 1. Changes in the $(A)$ relative water content and (B) osmotic potential in the middle leaves of in well-watered control (black bar) and water-deficit (grey bar) plants. The values are given with mean \pm SE for $\mathrm{n}=4$. * and $* *$ indicate significant differences between control and stress treatment at $p<0.05$ and $p<0.01$, respectively.

measured in water-deficit treated white clover(Lee et al., 2004).

Low RWC would be the consequence of low water uptake and hydraulic flow rates within the plants, or high rates of water loss (Bittman and Simpson, 1989). In many studies on stress physiology, $\Psi \pi$ has often been used as a criterion to define the intensity of stress that plants haves been exposed to. It has been widely reported that the decrease in RWC and $\Psi \pi$ is responsible for the decrease in photosynthetic $\mathrm{CO}_{2}$ assimilation (Lee et al., 2008a), nitrogen assimilation (Kim et al., 2004; Lee et al., 2005) and cell extension process (Katerji et al., 1997; Lee et al., 2007). These, in turn, lead to NADPH accumulation and subsequent leakage of electrons leading to enhance the generation of ROS (Foyer and Noctor, 2003; Lee et al., 2009b) and activate the production of defense-related proteins (Cavalcanti et al., 2004; Lee et al., 2009b) and compatible solutes (Benhamou, 1995; Lee et al., 2009a).

It has been also well documented that biotic and abiotic stresses result in an increase in cell lignification (Katerji et al., 1997; Lee et al., 2007) which has a significant influences on cell expansion, growth, nutrient content and digestibility in forage crops. Indeed, five days of water-deficit treatment changed the enzymatic activity of peroxidases (Fig. 2) and PPO and PAL (Fig. 3) in leaves with different maturity, leading a significant difference in total phenol and lignin concentration (Fig. 4). For the response of peroxidases 

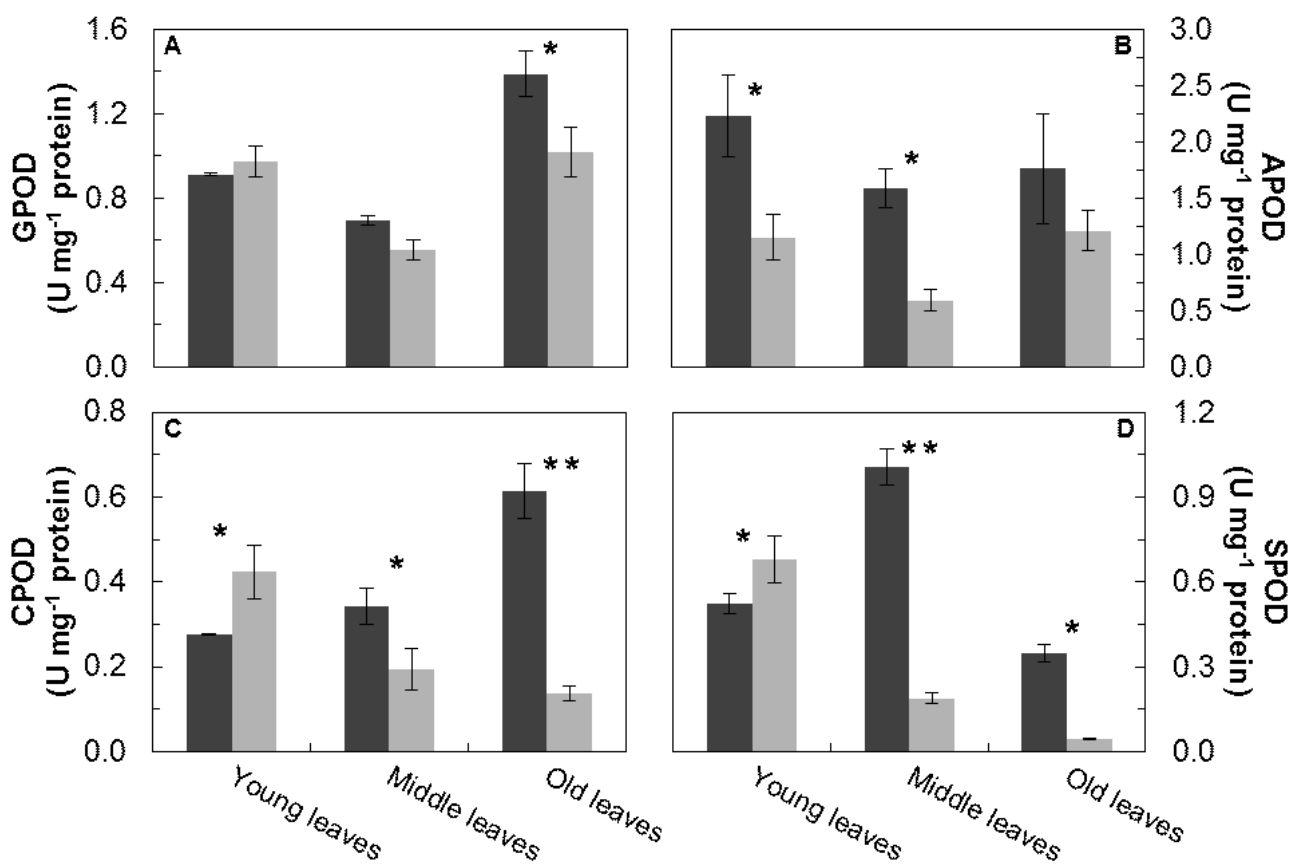

Fig. 2. Changes in the activities of (A) guaiacol peroxidase, GPOD, (B) ascorbate peroxidase, APOD, (C) coniferyl alcohol peroxidase, CPOD and (D) syringaldazine peroxidase, SPOD, in well-watered control (black bar) and water-deficit (grey bar) plants. The values are given with mean \pm SE for $n=4$. * and ** indicate significant differences between control and stress treatment at $p<0.05$ and $p<0.01$, respectively.

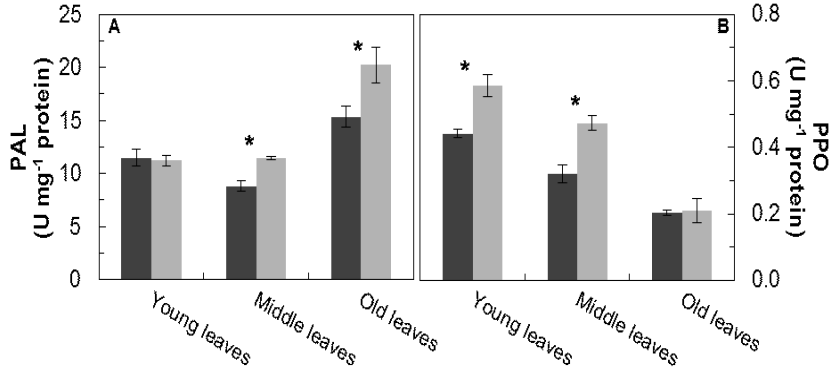

Fig. 3. Changes in the activities of $(A)$ phenylalanine ammonia-lyase, PAL and (B) polyphenol oxidase, $\mathrm{PPO}$, in well-watered control (black bar) and water-deficit (grey bar) plants. The values are given with mean $\pm \mathrm{SE}$ for $\mathrm{n}=4$. * and $* *$ indicate significant differences between control and stress treatment at $p<0.05$ and $p<0.01$, respectively.

activity to water-deficit treatment, the activity of four peroxidases examined in this study (GPOD, APOD, CPOD and SPOD) was depressed especially in middle and old leaves when compared with that of control leaves, respectively. In general, the isozymes of peroxidases participate in the modulation of cell wall properties during plant growth partly through catalyzing the formation of covalent

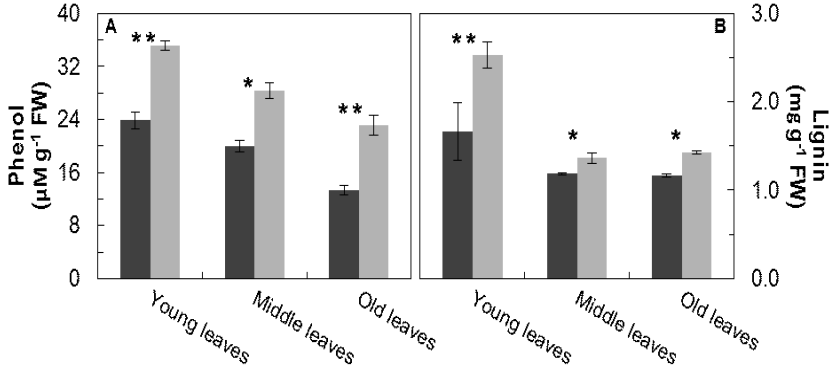

Fig. 4. Changes in the contents of $(A)$ phenol and $(B)$ lignin in well-watered control (black bar) and water-deficit (grey bar) rape plants. The values are given with mean $\pm \mathrm{SE}$ for $\mathrm{n}=4$. * and ** indicate significant differences between control and stress treatment at $p<0.05$ and $p<0.01$, respectively.

crosslinks after oxidation of ester- and ether-bound phenolic acids and also through the oxidative coupling of cinnamoyl alcohol moieties to generate lignin (Iiyama et al., 1994).

The results observed in middle and old leaves are thus inconsistent with those of previous study with white clover which showed a significant correlation between the increased activity of GPOD, CPOD and SPOD and the 
increase in lignin content (Lee et al., 2007). The discrepancy is mainly due to the difference in stress intensity exposed. Indeed, in white clover leaves, the enhanced activation of GPOD, CPOD and SPOD along with the increase in lignin concentration occurred when leaf water potential was -2.27 or less (from $21 \mathrm{~d}$ after water-deficit treatment). Additionally, less or limited activation in matured and old leaves when compared to white clove leaves might be associated with leaf cell wall property of leaves examined in two experiments. For examples, the middle and old leaves of rape plants has already higher level of complex net of crosslinking among monolignols, proteins and polysaccharides leading to limit the activation of cell wall peroxidases (Iiyama et al., 1994) compared to white clover leaves. On the other hand, in young leaves, a significant increase in CPOD (+34\%) and SPOD (+24\%) activity as affected by water-deficit treatment was apparent (Fig. 2C and D). This result indicate that young leaves (less cross-link and higher plasticity) are more susceptible to water deficit and have the higher catalytic activity for coniferyl alcohol and syringaldazine, the analogues of lignin monomer. Quiroga et al. (2000) reported that both cationic and anionic peroxidases showed the highest catalytic efficiency when the analogue of lignin monomer was used as substrate for peroxidases. In addition, CPOD and SPOD activities were also closely related total phenol content (Lee et al., 2007).

Water-deficit resulted in an activation of phenylalanine ammonia-lyase (PAL) activity in middle and old leaves (Fig. 3A). PAL is involved in the conversion of L-phenylalanine to trans-cinnamic acid, a key intermediate in the pathway of phenolics and lignin (Rivero et al., 2001). Therefore, in this study, enhance activity of PAL is responsible to the increase of phenol and lignin concentration (Fig. 4A and B). Polyphenol oxidase (PPO) is considered to catalyze the oxidation of polyphenols and hydroxylation of monophenols (Rivero et al., 2001) and lignification of plant cells (Lee et al., 2007). Several studies have indicated that phenoloxidizing enzymes may participate in response to the defense reaction and hypersensitivity to biotic and abiotic stress (Jouili and El Ferjani, 2003; Jung, 2004). It thus can be suggested that the increased phenol and lignin concentration in water-deficit stressed leaves would be attributed to the activation of PPO.
In this study, $5 \mathrm{~d}$ of water-deficit treatment increased total phenol concentration by $48 \%, 39 \%$ and $73 \%$ (Fig. 4A), and lignin concentration by $51 \%, 15 \%$ and $22 \%$ (Fig. 4B), respectively, in young, middle and old leaves. These results indicate that lignification process in forage rape plants occurs, but that the responses of peroxidases and lignifying enzymes were different depending on leaf maturity.

\section{CONCLUSION}

Short term treatment of water-deficit for $5 \mathrm{~d}$ results in a significant decrease of leaf water status. This was accompanied with changes in the activity of peroxidases and lignifying-related enzymes. GPOD, APOD, CPOD and SPOD were depressed or not changed in middle and old leaves, whereas CPOD and SPOD were significantly activated in young leaves. The activation of PAL and PPO was observed in middle and old leaves for PAL and in young and middle leaves for PPO. These results suggest that peroxidases in middle and old leaves did not involve in lignification under mild water-deficit stress, whereas CPOD and SPOD in young leaves participate in lignification by a coordination with PAL and PPO to incorporate phenol and lignin into the cell walls. The works further will be necessary to assess whether severe drought would be responsible for the structural rigidity and lignin incorporation in middle and old leaves, which lead to a reduction of growth and digestibility in forage rape plants.

\section{ACKNOWLEDGEMENTS}

This study was financially supported by Chonnam National University, 2012.

\section{REFERENCES}

Benhamou, N. 1995. Immunocytochemistry of plant defense mechanisms induced upon microbial attack. Microscopy Research and Technique. 31:63-78.

Bittman, S. and Simpson, G.M. 1989. Drought effects on water relations of three cultivated grasses. Crop Science. 29:992-999.

Cavalcanti, F.R., Oliveira, J.T.A., Martins-Miranda, A.S., Viegas, R.A. and Silveira, J.A.G. 2004. Superoxide dismutase, catalase and peroxidase activities do not confer protection against oxidative 
damage in salt-stressed cowpea leaves. New Phytologist. 163: 563-571.

D'Cunha, G.B., Satyanaraan, V. and Nair, P.M. 1996. Stabilization of phenylalanine ammonia-lyase containing Rhodotorula glutinis cells for the continuous synthesis of phenylalanine methyl ester. Enzyme and Microbial Technology. 19:421-427.

Foyer, C.H. and Noctor, G. 2003. Redox sensing and signaling associated with reactive oxygen in chloroplasts, peroxisomes and mitochondria. Physiologia Plantarum. 119:355-364.

Iiyama, K., Lam, T.B.T. and Stone, B.A. 1994. Covalent cross-links in the cell wall. Plant Physiology. 104:315-320.

Jouili, H. and El Ferjani, E. 2003. Changes in antioxidant and lignifying enzyme activities in sunflower roots (Helianthus annuus L.) stressed with copper excess. Comptes Rendus Biologies. 326: 639-644

Jung, S. 2004. Variation in antioxidant metabolism of young and mature leaves of Arabidopsis thaliana subjected to drought. Plant Science. 166:459-466.

Katerji, N., van Hoorn, J.W., Hamdy, A., Mastrorilli, M. and Mou Karzel, E. 1997. Osmotic adjustment of sugar beets in response to soil salinity and its influence on stomatal conductance, growth and yields. Agricultural Water Management. 34:57-69.

Kim, T.H., Lee, B.R., Jung, W.J., Kim, K.Y., Avice, J.C. and Ourry, A. 2004. De novo protein synthesis in relation to ammonia and proline accumulation in water stressed white clover. Functional Plant Biology. 31:847-855.

Lee, B.R., Jin, Y.L., Avice, J.C., Cliquet, J.B., Ourry, A. and Kim, T.H. 2009a. Increased proline loading to phloem and its effects on nitrogen uptake and assimilation in water-stressed white clover (Trifolium repens). New Phytologist. 182:654-663.

Lee, B.R., Jin, Y.L., Jung, W.J., Avice, J.C., Morvan-Bertrand, A., Ourry, A., Park, C.W. and Kim, T.H. 2008a. Water-deficit accumulates sugars by starch degradation--not by de novo synthesis--in white clover leaves (Trifolium repens). Physiologia Plantarum. 134:403-411.

Lee, B.R., Jung, W.J., Kim, K.Y., Avice, J.C., Ourry, A. and Kim, T.H. 2005. Transient increase of de novo amino acid synthesis and its physiological significance in water-stressed white clover. Functional Plant Biology. 32: 831-838.

Lee, B.R., Jung, W.J., Lee, B.H., Avice, J.C., Ourry, A. and Kim, T.H. 2008b. Kinetics of drought-induced pathogenesis-related proteins and its physiological significance in white clover leaves. Physiologia Plantarum. 132:329-337.

Lee, B.R., Kim, K.Y., Jung, W.J., Avice, J.C., Ourry, A. and Kim, T.H. 2007. Peroxidases and lignification in relation to the intensity of water-deficit stress in white clover(Trifolium repens L.). Journal of Experimental Botany. 58: 1271-1279.

Lee, B.R., Li, L.S., Jung, W.J., Avice, J.C., Ourry, A. and Kim, T.H. 2009b. Water deficit-induced oxidative stress and the activation of antioxidant enzymes in white clover leaves. Biologia Plantarum. 53:505-510.

Lee, B.R., Muneer, S., Park, S.H., Zhang, Q. and Kim, T.H. 2013. Ammonium-induced proline and sucrose accumulation, and their significance in antioxidative activity and osmotic adjustment. Acta Physiologae Plantarum. 35:2655-2664.

Quiroga, M., Guerrero, C., Botella, M.A., Barceló, A., Amaya, I., Medina, M.I., Alonso, F.J., Forchetti, S.M., Tigier, H. and Valpuesta, V. 2000. A tomato peroxidase involved in the synthesis of lignin and suberin. Plant Physiology 122:1119-1127.

Rivero, R.M., Ruiz, J.M., García, P.C., López-Lefebre, L.R., Sánchez, E. and Romero, L. 2001. Resistance to cold and heat stress: accumulation of phenolic compounds in tomato and watermelon plants. Plant Science. 160:315-321.

Sánchez, F.J., De Andres, E.F. Tenorio, J.L. and Ayerbe, L. 2004. Growth of epicotyls, turgor maintenance and osmotic adjustment in pea plants (Pisum sativum L.) subjected to water stress. Field Crops Research. 86:81-90.

Singleton, V.L. and Rossi, J.A. 1965. Colorimetry of total phenolic with phosphomolybdicphosphotungstic acid reagents. American Journal of Enology and Viticulture. 16:144-158.

Siriphanich, J. and Kader, A.A. 1985. Effects of $\mathrm{CO}_{2}$ on cinnamic acid 4-hydroxylase in relation to phenolic metabolism in lettuce tissue. Journal of the American Society for Horticultural Science. 110:333-335

Souza, R.P., Machado, E.C. Silva, J.A.B. Lagôa, A.M.M.A. and Silveira, J.A.G. 2004. Photosynthetic gas exchange, chlorophyll fluorescence and some associated metabolic changes in cowpea (Vigna unguiculata) during water stress and recovery. Environmental and Experimental Botany. 51:45-56.

(Received November 29, 2013 / Revised February 12, 2014 / Accepted February 14, 2014) 